Mother-child engagement in sports and outdoor activities: Intensive mothering, purposive leisure, and implications for health and relationship closeness

\author{
Chris Knoester* \\ Ohio State University \\ Victoria T. Fields \\ Eastern Michigan University
}

*Please direct all correspondence to Chris Knoester, 152 Townshend Hall, 1885 Neil Ave, The Ohio State University, Columbus, OH 43210 (knoester.1@ osu.edu), Ph: 614-247-7261 (work), 614-725-0075 (home), fax: 614-292-6687.

**This is a peer-reviewed preprint of the article:

Knoester. Chris, and Victoria Fields. 2020. "Mother-child Engagement in Sports and Outdoor Activities: Intensive mothering, purposive leisure, and implications for health and relationship closeness." International Review for the Sociology of Sport 55(7):933-952. First Copyright (c) [2019] (Sage). DOI: https://doi.org/10.1177\%2F1012690219855916. 


\begin{abstract}
Using Fragile Families \& Child Well-Being data $(\mathrm{N}=3,252)$ from the U.S., this study examines mother-child interactions in sports and outdoor activities with their nine-year old children, and their association with mothers' perceptions of the extent to which they think they are a good parent. The study also considers the implications of these mother-child engagement activities for the health of both generations and for their feelings of relationship closeness. The results reveal that most mothers participate in sports or outdoor activities with their child once per week or more; also, mother-child interactions are positively associated with mothers' perceptions of being a good parent. In addition, we find that mother-child interactions in sports and outdoor activities are positively associated with mothers' reports of their health and relationship closeness, but that it is children's organized sports participation (and not motherchild interactions in sports and outdoor activities) that is positively associated with the children's reports of their health and mother-child relationship closeness. Overall, there is support for understanding mother-child interactions in sports and outdoor activities as extensions of intensive mothering expectations and purposive leisure goals, and interactions that have positive implications for health and relationship closeness.
\end{abstract}




\section{Mother-Child Engagement in Sports and Outdoor Activities: Intensive Mothering, Purposive Leisure, and Implications for Health and Relationship Closeness}

Cultures of intensive mothering and expectations for "good" parenting encourage motherchild interactions in sports and outdoor activities. In part, this is because there are assumed benefits that are expected to derive from such interactions, such as improved health and closer relationship bonds (Harrington, 2015; Hays, 1996; Shaw and Dawson, 2001). Yet, there is little evidence of how frequently these types of mother-child interactions occur, much less their implications for health and relationship closeness.

Research has shown that physical activity is associated with positive health outcomes throughout the life course that include reductions in chronic diseases, hypertension, osteoporosis, diabetes, high blood pressure, obesity, and cholesterol risks-- as well as enhanced functional capacity (Erkelenz et al., 2014; Haskell et. al., 2009; Hesketh et. al, 2014; Humphryes et. al, 2014; Rhodes, et. al., 2013). Thus, mother-child engagement in sports and outdoor activities can be beneficial for the health statuses of both generations, since it may offset sedentary time and incur at least moderate levels of physical activity (Booker et al., 2015; Kahan \& McKenzie, 2015; Project Play, 2016). Furthermore, coactivity can help facilitate, support, and model continued commitments to sports and outdoor activities (Harrington, 2015; Rhodes and Lim, 2018). Yet, there is a lack of evidence of the extent to which mother-child interactions in sports and outdoor activities occur, as well as their implications for the health of both generations.

Mother-child engagement in sports and outdoor activities may also bring about interpersonal benefits, such as relationship closeness (Harrington, 2015; Rhodes and Lim, 2018; Shaw and Dawson, 2001). This is important to study because mothers are encouraged and expected to be nurturers (Bianchi et al., 2006; Messner, 2009; Risman, 1998). Relatedly, mothers 
spend markedly more time with their children than do fathers through direct interactions, accessibility, and various forms of care (Bianchi et al., 2006; Craig and Mullan, 2010; Phares et. al, 2009). Although mothers hold the majority of the caretaking responsibilities for children in families, they also allocate their time to having playful interactions with their children (Rhodes et. al., 2013; Shaw and Dawson, 2001). Thus, although fathers are often seen as experts on sports and outdoor activities, and typical father-child interactions are more focused on sports than are typical mother-child interactions, mother-child interactions in sports and outdoor activities are expected to be common (Coakley, 2006; Hays, 1996; Messner, 2009; Shaw and Dawson, 2001). However, there is also a paucity of evidence of how frequently mother-child interactions in sports and outdoor activities occur, as well as their implications for relationship closeness.

Consequently, the purpose of this study is to analyze mother-child interactions in sports and outdoor activities among a birth cohort of nine-year old children from large U.S. cities. Besides offering descriptive analyses of the patterns and predictors of these interactions, we also examine the implications of these mother-child interactions for notions of good parenting, the health statuses of both generations, and the closeness of mother-child relationships. A focus on mother-child interactions with their nine-year-old children is especially valuable because children appear to decrease their involvement in sports and outdoor activities as they approach adolescence; also, children become increasingly independent from their mothers (Knoester et al., 2006; Project Play, 2016; Raley et al., 2012; Sabo and Veliz, 2008).

The current study advances research in a number of ways. First, it provides a muchneeded assessment of how mothers engage with their (nine-year-old) children in sports and outdoor activities. There is a vast literature on the frequency and implications of physical activity over the life course, but far less information on a variety of commonly shared mother-child 
activities that are alternatives to indoor and passive uses of time-especially from a quantitative perspective (Booker et al., 2015; Haskell et al., 2009; Messner and Musto, 2014; Rhodes and Lim, 2018; Shaw and Dawson, 2001). Second, there is an emphasis on organized youth sports activities, but little research on a variety of other more informal pursuits that are encompassed by sports and outdoor activities (Coakley, 2011; Messner and Musto, 2014; Project Play, 2016). Finally, our research analyzes mother-child sports and outdoor activity interactions among disadvantaged families (e.g., low socioeconomic, unmarried, and non-white families) as opposed to a traditional focus on parenting and mother-child relationships within middle-class, married, and white families (Messner, 2009; Shaw and Dawson, 2001; Solomon-Moore et al., 2017; Wheeler and Green, 2014). Thus, we can complement and extend extant notions and evidence of the extent to which mother-child interactions in sports and outdoor activities occur, and their implications for perceptions of good parenting, health, and relationship closeness.

In fact, the data are unique because they follow a large sample of disproportionately disadvantaged (i.e., low SES, racial/ethnic minority, unmarried parents) U.S. families over many years. Yet, because the focus in collecting information is on the biological mothers and fathers, and their new child, the structure of the data assumes that parents are heterosexual and that biological mothers and fathers are likely to co-parent. In addition, much of the conceptual framework for the present study builds off of gendered notions of parenting and the relative expectations that are constructed, relayed, and encouraged to mothers and fathers-expectations that primarily have served to benefit men (Hays, 1996; Risman, 1998). Thus, it is important to recognize from the onset that gender normative assumptions and heteronormativity are embedded within the present study and that follow-up work is needed to assess the extent to 
which the conceptual framework and results from the study are applicable to all families, including those with same-sex parents and/or no biological mother present, for example.

\section{Conceptual Framework}

The conceptual framework for this study draws from role theory (Biddle, 1986; Goode, 1960) to better understand how parents understand what they should do and how this may affect their notions of good parenting, their parenting behaviors, and the consequences of their parenting behaviors. Role theory recognizes that status positions in social structure, such as being a parent, are accompanied by role expectations for how one should think and behave. Role expectations are learned and actively negotiated, largely through social interactions. Role expectations for parents, also known as parenting expectations, are thus important factors for how parents think that they should parent, how they feel about how well they are parenting, and the parenting behaviors that they engage in —with important consequences for family health and well-being (Coakley, 2006; Hays, 1996; Knoester and Petts, 2017; Milkie et al., 2015).

The particular emphasis of this study is a consideration of how parenting role expectations for intensive mothering and purposive leisure may promote mother-child engagement activities in sports and outdoor activities, with implications for both generations' feelings of relationship closeness and subjective health. We expect that intensive mothering and

purposive leisure expectations largely encourage mother-child interactions in sports and outdoor activities because they recognize mothers as primary caregivers who should proactively shape their family routines in ways that will maximize child development and family well-being (Elliott et al., 2015; Hays, 1996; Shaw and Dawson, 2001).

Nonetheless, before proceeding, it is important to note that mothering behaviors are frequently coordinated with, or otherwise shaped by, parenting role expectations for fathers and 
actual fathering behaviors (Bianchi et al., 2006; Craig and Mullan, 2010; Hays, 1996; Marsiglio and Roy, 2012; Raley et al., 2012). Indeed, "new" fatherhood ideals have increasingly encouraged fathers to become more involved in their children's lives and many fathers have responded to these fathering role expectations by becoming especially involved in their children's lives - at least compared to previous generations of fathers. Father-child interactions in sports and outdoor activities are particularly common, as men have long been passionate about, and comfortable with, these types of interactions (Author Citation; Coakley, 2006; Craig and Mullan, 2010; Knoester and Petts, 2017; Milkie et al., 2015). Thus, role expectations for fathering, as well as fathering behaviors, are integral to our conceptual framework and analysis. They both also seem to have become heightened in recent decades, along with mothering expectations and behaviors (Author Citation; Bianchi et al., 2006; Coakley, 2006). Nonetheless, because of gendered parenting expectations and experiences, inequalities that have led to the continued pattern of mothers spending at least twice as much time as fathers in being engaged with their children, and the attention that has been focused on father-child interactions in sports and outdoor activities, we reserve our attention in this study for mothering expectations and behaviors, and utilize measures of fathering behaviors as control variables (Author Citation; Bianchi et al., 2006; Coakley, 2006; Craig and Mullan, 2010; Milkie et al., 2015),.

\section{Intensive Mothering}

Intensive mothering refers to heightened parenting role expectations that have emerged for mothers to overwhelmingly prioritize their child's emotional, physical, social, and academic development- nearly at all cost (Hays, 1996; Lareau, 2003; Stone, 2007). Mothers have traditionally acted as the primary caretakers for their children and so the expectations seem in line with traditional roles (Hays, 1996; Risman, 1998). However, intensive mothering has 
especially appeared to take hold over recent decades; that is, it has increased since the middle of the $20^{\text {th }}$ century, at least. One factor seems to be the rise in technological advancements, and sometimes the ability to contract work out, which have allowed domestic work to be done more efficiently. Another factor seems to be the trend towards lower fertility and an emphasis on quality over quantity in childrearing. Relatedly, mothers-- and the population as a whole-- have become more educated and pursued strategic ways to boost child development, often with concerns about safety, future opportunities, and competitive advantages in mind. In fact, an influx in research evidence and advice from child-rearing experts, such as pediatricians, have encouraged mothers to attempt to live up to intensive mothering expectations by recognizing parenting as a science. On balance, research, internalized mothering expectations, and parenting recommendations have tended to result in mothers feeling that they should spend even more time with their children, compared to years past (Cherlin, 2014; Hays, 1996; Stone, 2007). Mothers seem to have followed these guidelines, at least based on trends in the amount of time that they spend with their children_-despite enhanced paid work commitments (Bianchi et al., 2006; Craig and Mullan, 2010; Milkie et al., 2015).

Nevertheless, intensive mothering expectations encourage mothers to do all that they can, and never be satisfied, in nurturing their sacred child's development and opportunities. Despite increased expectations for "new" and engaged fathering, mothers continue to spend markedly more time with their children, compared to fathers (Bianchi et al. 2006; Craig and Mullan, 2010; Raley et al., 2012). Consequently, the culture of intensive mothering has served to maintain gendered inequalities in parenting (Bianchi et al., 2006; Craig and Mullan, 2010; Hays, 1996; Raley et al., 2012; Risman, 1998). Indeed, intensive mothering is not a paid endeavor and fathers 
are rarely willing or expected to give up career opportunities to pursue intensive parenting duties (Hays, 1996; Risman, 1998; Raley et al., 2012; Stone, 2007).

Intensive mothering expectations have also seemed to have especially taken hold in middle and upper-class, well-educated, and married parent households; nonetheless, the majority of working class, less-educated, and single parent families also recognize them and frequently view them as aspirational (Elliott et al., 2015; Hays, 1996; Lareau, 2003; Stone, 2007). However, intensive mothering is enabled by financial support from a co-parent and privileged social structural locations (Christopher, 2012; Hays, 1996; Kendig and Bianchi, 2008; Stone, 2007). Thus, married, white, and high SES mothers spend more time with their children, including time in sports and outdoor activities, than do single, racial-ethnic minority, and low SES mothers (Kendig and Bianchi, 2008; Sabo and Veliz, 2008). Overall, intensive mothering expectations are important to study not only because they direct parent-child interactions and have implications for inequalities and social mobility, but also because they amplify work-family conflicts and parenting strains (Hays, 1996; Lareau, 2003; Stone, 2007).

\section{Purposive Leisure}

We assume that intensive mothering expectations are likely to combine with parenting role expectations for purposive leisure in affecting mother-child interactions in sports and outdoor activities. Purposive leisure refers to strategic decisions that parents make about the activities that they encourage - especially during times of leisure. Family interactions that surround sports and outdoor activities are popular and well-respected choices, because of their tendency to bring enjoyment, physical activity, and family bonding experiences (Harrington, 2015; Sabo and Veliz, 2008; Shaw and Dawson, 2001). Oftentimes, they are even seen as investments (Coakley, 2006; 2011; Eckstein, 2017; Messner, 2009). 
A rise in recognizing and promoting sports and outdoor activities appears to have occurred largely in step with the increased emphasis on intensive mothering over recent decades. That is, especially over the past 60-70 years in the U.S., the importance of physical activity, fitness, and sports involvement has become prioritized by public policies, education, and family interactions. This is evidenced by a growth in formal and informal physical education, fitness experts, and sports involvement (Coakley, 2006; Messner, 2009; Montez de Oca, 2005). Substantially, the focus has been on the benefits of sports and outdoor activities for children (Coakley, 2006; Eckstein, 2017; Messner, 2009; Project Play, 2016). Thus, promoting sports and outdoor activities has become a hallmark expectation for "good" parenting. Children from all backgrounds are expected to benefit from sports and outdoor activities, largely because of the health benefits and quality social relationships that are assumed to emerge (Coakley, 2006; 2011; Eckstein, 2017; Messner, 2009; Project Play, 2016; Wheeler and Green, 2014).

Many parents are mindful of the health and family interactions that family-centered sports and outdoor activities can bring. In fact, they tend to encourage sports and outdoor activities, including family-based coactivity, because they believe that they are likely to lead to improved health and relationship closeness among family members (Shaw and Dawson, 2001; Harrington, 2015). There is substantial research that supports positive associations between sports activities and health; similarly, there is evidence that sports participation is positively associated with the quality of family relationships (Project Play, 2016; Sabo and Veliz, 2008). Related to these benefits, and the increased motivation to encourage coactivity in sports and outdoor activities, is the evidence that coactivity encourages each generation to participate more than they may have otherwise and to continue commitments to sports and outdoor activities, over the life course (Project Play, 2015; Rhodes and Lim, 2018). 
Indeed, a mother's encouragement, modelling of an active lifestyle, and active involvement in their child's sporting activities are found to be key factors in encouraging familybased participation in physical activity (Project Play, 2016; Wheeler and Green, 2014). Specifically, physical activity has the potential to improve the health and overall quality of life for mothers and their families (Dlugonski \& Motl, 2013). Also, family members who often played together report higher levels of happiness, healthy functioning, and unity because the shared activities provide an environment for open communication, interaction, and problem solving (Burns and Grafe, 2006). Therefore, in terms of family priorities, mothers rank physical activity almost as high as homework and far higher than other activities (Rhodes et. al., 2013). Mothers appear to recognize the benefits of participating in sport and outdoor activities and are enthusiastic about creating family-based interactions around them (Rhodes and Lim, 2018; Wheeler and Green, 2014). There is some evidence that encouraging sports and outdoor activities is more common among higher socioeconomic status families, however (Lareau, 2003; Project Play, 2016; Wheeler and Green, 2014). This is consistent with claims that more welleducated and middle-class mothers are especially likely to be mindful of the benefits of purposive leisure and to have the resources and time-availability to enact intensive mothering around sports and outdoor activities. Thus, disadvantaged families may be less likely to benefit from the positive effects of coactivity in sports and outdoor activities (Harrington, 2015; Hays, 1996; Messner, 2009; Project Play, 2016; Stone, 2007).

\section{Background Characteristics}

Indeed, in analyzing mother-child engagement activities in sports and outdoor activities and their implications for health and relationship closeness, several factors are important to 
consider. These factors include background characteristics such as socioeconomic status, race/ethnicity, family structure, gender, and other factors.

Socioeconomic status is expected to be positively associated with mother-child interactions in sports and outdoor activities and their reports of health (Cairney et al., 2015; Project Play, 2016; Ramraj et al., 2016; Sabo and Veliz, 2008). SES also shapes parenting practices and thus may be tied to relationship closeness, as well (Harrington, 2015; Lareau, 2003; Sabo and Veliz, 2008). In addition, there may be unique racial-ethnic patterns in mother-child interactions in sports and outdoor activities, with associated health and relationship closeness differences (Brooks et al., 2017; Project Play, 2015; Ramraj et al., 2016; Sabo and Veliz, 2008).

Family structure may also affect mother-child interactions in sports and outdoor activities and be linked to health and relationship closeness (Althoff et al., 2017; Marsiglio and Roy, 2012; Project Play, 2015; Sabo and Veliz, 2008). Specifically, single parents may have more individual responsibilities, and stressors that arise from these responsibilities, but also more influence as a primary caregiving parent (Elliott et al., 2015; Kendig and Bianchi, 2008). Also, father-child interactions in sports and outdoor activities are expected to be linked to patterns of mother-child interactions (Author Citation; Coakley, 2006; Marsiglio and Roy, 2012; Sabo and Veliz, 2008).

In terms of gender, there are structures and cultures in place that disproportionately encourage males to participate in sports and outdoor activities throughout the life course, compared to females (Messner, 2009; Project Play, 2015). In addition, same-sex coactivity seems to be more common and valued than cross-sex coactivity-- especially for males (Cairney et al., 2015; Messner, 2009; Sabo and Veliz, 2008). Other relevant factors include age, family size, previous levels of health, and participation in organized sports (Althoff et al., 2017; Cairney et al., 2015; Physical Activity Council, 2016; Sabo and Veliz, 2008). 


\section{Hypotheses}

Our conceptual framework and previous research on intensive mothering, purposive leisure, and the health and relationship implications of sports and outdoor activities suggest: Hypothesis \#1: Mother-child interactions in sports and outdoor activities occur frequently, and will be positively associated with mothers' perceptions of being a good parent.

Hypothesis \#2: Socioeconomic status will be positively associated with mother-child interactions in sports and outdoor activities.

Hypothesis \#3: Mother-child interactions in sports and outdoor activities will be positively associated with reports of mothers' and children's health statuses.

Hypothesis \#4: Mother-child interactions in sports and outdoor activities will be positively associated with mothers' and children's reports of their relationship closeness.

\section{Method}

Our data derive from the Fragile Families and Child Wellbeing Study (FFCW). The FFCW is a longitudinal birth cohort study of the families of 4,898 children born in the U.S. between 1998 and 2000. It is useful for the purposes of the present study because it offers detailed information on family demographics, interactions, relationships, and well-being indicators. The FFCW contains high percentages of low-income, non-white, and unmarried parents, as the sample was drawn from hospitals in 20 cities with populations over 200,000 (Reichman et al. 2001). Parents were interviewed right after a birth (W1) and the families have been followed, and interviewed periodically (e.g., $\mathrm{W} 2=1$ year, $\mathrm{W} 3=3$ year, $\mathrm{W} 4=5$ year, $\mathrm{W} 5=$ 9 year, $\mathrm{W} 6=15$ year), since then .

We primarily use data from when the child was 9 years old (W5), from interviews conducted between 2007-2010, since that is when questions about mother-child interactions in 
sports and outdoor activities are first asked, children first report on family relationships, and research suggests that children are especially likely to be involved in sports and outdoor activities (Project Play, 2016; Sabo and Veliz, 2008). Our sample consists of the 3,252 mothers who reported on mother-child interactions in sports and outdoor activities and who lived with their child all or most of the time at W5; these mothers were considered primary caregivers of the focal child, in the FFCW (93\% of all mothers interviewed).

\section{Variables}

Our primary variables are W5 reports of engagement in sports and outdoor activities, self-ratings of parenting quality, perceptions of overall health, and assessments of mother-child relationship closeness. Background characteristics are used as control variables when analyzing the connections between mother-child interactions, health indicators, and relationship closeness. Descriptive statistics for all variables are displayed in Table 1.

\section{(Table 1 about here)}

Three variables indicate engagement in sports or outdoor activities. Our focus is on mothers' W5 reports of mother-child interactions in sports or outdoor activities. This measure indicates how frequently mothers engaged in sports or outdoor activities with their child in the month prior to the interview $(0=$ never, $1=1-2$ times in the past month, $2=1 x /$ week, $3=$ several times/week, 4 =daily). We predict variance in this variable, with a focus on socioeconomic status as the independent variable in this particular model, but primarily consider it as our main independent variable. We also utilize mothers' W5 reports of children's participation in organized athletic activities outside of school hours in the past year $(1=y e s)$, in an attempt to consider a child's organized sports experiences. Finally, as a complement to understanding the significance of mother-child engagement, we include a measure of father-child interactions in 
sports and outdoor activities in the last month $(0=$ never, $4=$ daily $)$, based on the highest level of interaction reported between the child and either the father at baseline or a mother's (new) romantic partner.

We use a W5 self-rating of parenting quality as our initial dependent variable, so that we can assess the extent to which mother-child engagement predicts whether mothers think they are doing a good job of being a parent $(1=$ not a very good parent, $2=a$ good parent, $3=a$ very good parent, 4 = an excellent parent). We anticipate that mothers who are more engaged with their child in sports and outdoor activities will believe that they are doing a better job as a parent.

Reports of a mother's and their child's health and relationship closeness are our primary dependent variables, when considering the implications of mother-child engagement in sports and outdoor activities. Mother's overall health is taken from responses to the question: "In general, how is your health?" $(1=$ poor, $2=$ fair, $3=$ good, $4=$ very good, $5=$ excellent $)$. Responses to a similar question, asked of the child, are used to indicate child's overall health (1 $=$ poor $;=$ excellent ). In addition, we utilize the W4 indicators of father's and child's overall health in our analyses because sports and outdoor activities as well as overall health can depend on prior levels of health. These are taken from mothers' reports. Mother's and child's reports of relationship closeness to one another are our final dependent variables. The measures are responses to questions that ask $(1=$ not very close, $2=$ fairly close, $3=$ quite close, $4=$ extremely close): "How close do you feel to ___ ? ? We use both mothers' and children's reports for our relationship closeness measures.

Background characteristics that may be linked to our primary variables, and that mostly act as control variables in our analyses, include socioeconomic status, race-ethnicity, family structure, gender, and other factors. Mother's educational attainment (coded with dummy 
variables indicating less than high school, high school, some college, or college or more educational attainment) and household income (in $\$ 10,000$ s) reflect socioeconomic status. For race-ethnicity, we consider the implications of mothers identifying as Black $(1=y e s)$ or (nonBlack) Latina $(1=y e s)$, with White/Other used as the reference category. Family structure measures are coded based on whether mothers are married (reference category), cohabiting, or living apart from the father. There is also an indicator to signify if the mother has a partner, who is not the (original) father, residing in the household $(1=y e s)$. Other factors include mother's age and child's gender $(1=$ female $)$.

\section{Results}

In attempting to test our hypotheses, our analysis involves assessing the patterns of mother-child engagement activities in sports and outdoor activities, using mother-child engagement to predict self-ratings of parenting, focusing on the extent to which socioeconomic status is associated with mother-child engagement in sports and outdoor activities, and then considering the extent to which engagement in sports and outdoor activities predicts health and relationship closeness. Specifically, we first use descriptive statistics to examine how often mother-child engagement in sports and outdoor activities occur. Then, we use ordinary least squares (OLS) regression analyses to predict self-ratings of parenting, mother-child engagement, the health of both generations, and relationship closeness. We include one logistic regression model in our analysis in order to predict children's participation in organized sports, for comparison purposes when considering the factors that predict mother-child engagement in sports and outdoor activities.

First, we scrutinize reports of mother-child engagement activities and other descriptive statistics. As shown in Table 1, mothers reported participating with their child in sports and 
outdoor activities about one time per week in the month prior to their interview, on average $(M=$ 1.92). In fact, $62 \%$ of mothers reported participating in this type of mother-child engagement for at least one time per week or more; $38 \%$ reported participating in these activities with their child several times per week or more (results not shown). Thus, there is support for our expectation that mother-child interactions in sports and outdoor activities occur frequently. By comparison, father-child interactions in sports and outdoor activities $(M=1.67)$ were reported to occur less frequently (paired $t$-test comparison for gender difference significant at $p<.001$ ). Also, it is notable that $50 \%$ of the children participated in organized athletics, over the past year. As expected from the sampling approach for the FFCW, the descriptive statistics also indicate that the mothers are disproportionately less-educated, poorer, racial-ethnic minority, and unmarried parents, compared to the general U.S. population (Reichman et al., 2001).

Second, we turn to analyzing the extent to which mother-child engagement in sports and outdoor activities is associated with self-perceptions of good parenting. As shown in Table 2, there is evidence that mother-child engagement is associated with self-ratings of being a good parent $(b=.06, p<.001)$. That is, more frequent mother-child interactions in sports and outdoor activities seem to have increased the likelihood that mothers would identify themselves as a very good or excellent parent. Thus, we find support for our expectation that mother-child engagement in sports and outdoor activities is seen as a hallmark of good parenting, through meeting parenting role expectations, as well.

Third, we predict the frequency with which mothers engage in sports or outdoor activities with their children. As shown in Model 1 of Table 3, in contrast to expectations, there is no evidence that socioeconomic status is positively associated with mother-child engagement in sports and outdoor activities. However, there is evidence that mothers who are white, single 
parents, have more children, and have daughters are more frequently engaged in sports and outdoor activities with their child. Father-child interactions and child's organized sports participation are also positively associated with mother-child engagement. Older mothers, racial-ethnic minority mothers, and mothers with a new partner are less frequently engaged in sports and outdoor activities with their child. Sensitivity analyses revealed that although socioeconomic status was positively associated with mother-child engagement in more basic models, the additions of W4 health statuses, race-ethnicity, and other indicators of sports involvement (ie., father-child interactions, organized sport participation) rendered the association nonsignificant. Indeed, SES is positively associated with health, whiteness, and organized sports participation (Cairney et al., 2015; Project Play, 2016; Ramraj et al., 2016). Relatedly, in more basic models, single mothers reported lower levels of mother-child interactions in sports and outdoor activities compared to married mothers; as in previous research, single mothers appear to be more engaged with their children (only) after accounting for their disadvantaged social locations (Kendig and Bianchi, 2008; Sabo and Veliz, 2008).

For comparison purposes, we also explored the extent to which SES and other factors predict children's organized sports participation over the past year, according to mothers' reports. There is evidence that socioeconomic status is positively associated with children's organized sports participation, as shown in Model 2. It may be that the culture of intensive mothering primarily encourages directing and supporting organized sports activities, as opposed to a variety of mother-child sports and outdoor activities, along the lines of concerted cultivation parenting role expectations that emphasize institutionalized activities (Hays, 1996; Lareau, 2003; Wheeler and Green, 2014). That is, parents may respond to perceptions of a competitive landscape for social mobility, the increased privatization of recreation opportunities, and unequal 
family resources to try and give their children advantages through enrollment in organized sports (Eckstein, 2017; Lareau, 2003; Messner, 2009). It is also notable that in contrast to the direction of their associations with mother-child interactions in sports and outdoor activities, daughters are less likely to play organized sports $(O R=.56, p<.001)$ and family size is negatively associated with a child's likelihood of playing organized sports $(O R=.87, p<.001)$. Finally, it seems that both models suggest evidence of family-based cultures of sports and outdoor activities with correlations between mother-child interactions in sports and outdoor activities, father-child interactions of these sort, and child's organized sports participation. This is consistent with previous research about family cultures surrounding sports and physical activities, and their representation of purposive leisure expectations for family members (Wheeler and Green, 2014). We now turn to considering the implications of mother-child interactions in sports and outdoor activities for health; we hypothesized that mother-child interactions would be positively associated with the health of both generations. As shown in Model 1 of Table 4, there is evidence of a positive association between mother-child sports or outdoor activities and mother's health $(b$ $=.03, p<.05)$, as expected. In contrast, there is not a positive association between mother-child engagement and child's health shown in Model 2. Instead, it seems that children's organized sports participation $(b=.13, p<.01)$ is what is meaningful in affecting their own health.

Finally, we focus on using mother-child engagement in sports and outdoor activities to predict relationship closeness. We hypothesized that mother-child engagement would be positively associated with mother's and child's reports of their relationship closeness. As shown in Model 1 of Table 5, there is a positive association between mother-child engagement and mother's report of relationship closeness $(b=.04, p<.001)$, as expected. For child's report of mother-child closeness, mother-child engagement in sports and outdoor activities is 
nonsignificant, as shown in Model 2. However, child's organized sports participation is positively associated with child's report of relationship closeness $(b=.08, p<.01)$, suggesting that from a child's perspective, the parental support that goes into funding, providing transportation, and conversing about organized sports may be especially important in building parent-child relationship closeness. Parents may also view this support as a means of meeting parenting role expectations for intensive mothering, involved fathering, and purposive leisure (Messner, 2009; Sabo and Veliz, 2008; Thompson, 1999; Wheeler and Green, 2014).

\section{Discussion}

In this study, we utilized role theory (Biddle, 1986; Goode, 1960) to better understand how intensive mothering and purposive leisure expectations may shape mother-child interactions and their implications for both generations. We assessed the frequencies with which motherchild interactions in sports and outdoor activities occur, the extent to which this engagement is associated with self-ratings of good parenting, and the factors that predicted mother-child interactions in sports and outdoor activities. We also analyzed the implications of mother-child engagement in sports and outdoor activities for the health of both generations and for their perceptions of relationship closeness. We anticipated that mother-child coactivity in sports and outdoor activities would lead to higher levels of subjective health and mother-child relationship closeness from the perspective of both generations. Furthermore, we expected that mother-child interactions in sports and outdoor activities would be positively associated with self-perceptions of being a good parent. Also, we expected that socioeconomic status would be positively associated with mother-child coactivity and that this association would reflect a measure of advantage that having parents of a higher social class can offer to children. We review our hypotheses and contextualize our findings, below. 
Our first hypothesis anticipated that we would find evidence of frequent mother-child interactions in sports and outdoor activities and that these interactions would be positively associated with perceptions of being a good parent. Indeed, we found support for this hypothesis; over $60 \%$ of mothers reported at least weekly mother-child interactions in sports or outdoor activities, and nearly $40 \%$ reported having these interactions several times per week or more. Furthermore, the frequency of these interactions was positively associated with mother's selfratings of being a good parent, suggesting that intensive mothering expectations for purposive leisure that include sports and outdoor activities are being recognized by mothers (Hays, 1996; Harrington, 2015; Shaw and Dawson, 2001; Wheeler and Green, 2014). In fact, despite cultural assumptions that sports and outdoor activities are largely masculine pursuits and fathers' areas of expertise, mother-child interactions in sports and outdoor activities were more common than father-child interactions in sports and outdoor activities, in this sample of parents. This finding is consistent with previous research on gender differences in parent-child interactions, including parent-child interactions in sports and outdoor activities (Coakley, 2006; Milkie et al., 2015; Sabo and Veliz, 2008)

Second, we anticipated that a mother's socioeconomic status would be positively associated with mother-child interactions in sports and outdoor activities, based on the culture and appeal of intensive mothering expectations for especially well-educated and middle-class families (Harrington, 2015; Hays, 1996; Stone, 2007). Also, we thought that this pattern may indicate the ability of more advantaged families to find time and resources to invest in motherchild coactivity in sports and outdoor activities (Hays, 1996; Project Play, 2016; Sabo and Veliz, 2008). However, we found sparse evidence of this association when predicting mother-child interactions. This may reflect the widespread awareness of intensive mothering and purposive 
leisure expectations among all mothers, as well as the potential cost-effectiveness (e.g., at local parks, in one's yard) of engaging in mother-child interactions in sports and outdoor activities, as opposed to other activity and leisure pursuits. Yet it also hints at how families may have adapted to intensive parenting and purposive leisure expectations (Christopher, 2012; Elliott et al., 2015).

There was substantial evidence that a mother's socioeconomic status was positively associated with their child's organized sports participation over the past year. Thus, it seems that intensive mothering and purposive leisure expectations largely involve encouraging a child's organized sports participation rather than mother-child interactions in sports and outdoor activities per se. This finding appears to be consistent with parenting expectations of concerted cultivation and an emphasis on organized athletic pursuits (Eckstein, 2017; Lareau, 2003; Messner, 2009; Sabo and Veliz, 2008). Of course, organized sports may also be viewed as purposive leisure for children, since they may encourage physical activity and peer interactions while also functioning as a form of day care for children, an opportunity to obtain adult teaching, and a safe space for recreation, too (Coakley, 2011; Messner, 2009; Project Play, 2016; Sabo and Veliz, 2008). Organized sports also tend to require more barriers to entry for more disadvantaged families because of the need for fees and transportation requirements, for example (Project Play, 2015; 2016; Sabo and Veliz, 2008). Christopher (2012) refers to similar techniques of responding to intensive parenting expectations by strategizing about, investing in, and organizing appropriate opportunities for children as "extensive" rather than "intensive" mothering. Of course, fathers may also be involved in these decisions and corresponding investmentsespecially in sports endeavors (Author Citation; Coakley, 2006; Sabo and Veliz, 2008). Yet, it is important to recognize the innovation and pursuit of extensive mothering as an adaptation to address competing commitments to mother-child interactions (e.g., mother's work, single 
parenting strains) as well as an attempt to offer enriched environments for children (e.g., safe, supervised, teaching by experts).

Finally, we hypothesized that mother-child interactions in sports and outdoor activities would be positively associated with the health of both generations and with their reports of relationship closeness. We found consistent evidence that mother-child interactions in sports and outdoor activities are positively associated with mothers' reports of their health and of the closeness of their mother-child relationships. Thus, mother-child interactions in sports and outdoor activities appear to be meaningful to mothers and seem to bring the assumed benefits of purposive leisure in sports and outdoor activities (Harrington, 2015; Wheeler and Green, 2014). Mothers seem likely to benefit from mother-child coactivity not only because they feel better about their parenting, but also because they obtain some measure of health benefits and the emotional fulfillment of helping to nurture close mother-child relationships. In sum, mothers appear to benefit from fulfilling intensive mothering, purposive leisure, and child-nurturing role expectations (Hays, 1996; Risman, 1998; Stone, 2007; Wheeler and Green, 2014).

In contrast, we did not find support for our expectation that mother-child interactions in sports and outdoor activities would be positively associated with children's reports of health and mother-child relationship closeness. Instead, it was children's organized sports participation that was positively associated with children's health and perceptions of mother-child relationship closeness. This seems to suggest that mother-child interactions in sports and outdoor activities act, and are viewed, as more incidental activities when compared to a child's organized sports participation - at least in terms of their implications for a child's reports of their health and mother-child relationship closeness. Yet, the positive association between children's organized sports participation and children's reports of mother-child closeness points to the (other) ways 
that mothers often support their children's sports participation, meet parenting role expectations, and indirectly nurture their children's health and closer mother-child relationships, in the process (Messner, 2009; Sabo and Veliz, 2008; Thompson, 1999; Wheeler and Green, 2014). Coactivity through children's organized sports participation is also a way that more advantaged families may be better able to invest in their children than may more disadvantaged families_-because of the costs and resources needed to support organized sports participation, especially with the neoliberal shift to more privatized organized sports opportunities (Eckstein, 2017; Project Play, 2015; 2016). Thus, lower SES, racial/ethnic minority, and single parent families may disproportionately lack affordable and convenient opportunities (Project Play 2015; 2016; Sabo and Veliz, 2008).

Overall, this study improves our understanding of the sociology of sport, parenting behaviors, and the patterns and implications of mother-child interactions in sports and outdoor activities. In terms of theory, the study integrates core family sociology concepts of parenting role expectations, and specifically intensive mothering and purposive leisure expectations, into novel considerations of the why, how, and potential effects of mothering behaviors involving sports and outdoor activities. Indeed, the results are in line with many of our expectations regarding intensive mothering expectations and purposive leisure. Thus, they are meaningful in describing patterns, noting associations, and recognizing the presence and influence of parenting cultures and expectations. In particular, the results affirm the significance of mothering expectations and behaviors for parenting practices_-even those involving sports and outdoor activities, which have been traditionally thought of as male domains (Author Citation; Coakley, 2006; Messner, 2009). The results of this study are also unique in demonstrating that these expected dynamics, patterns, and apparent consequences for health and relationship closeness 
exist even within disadvantaged populations (Author Citation; Elliott et al., 2015). Thus, the realization of parenting role expectations and the potential benefits of mother-child interactions in sports and outdoor activities are widely applicable.

Nonetheless, despite the presence of a number of statistically significant findings in our analyses, it is important to note that the effect sizes for the findings are very modest. In part, this may be a function of the use of secondary data that are not designed to comprehensively analyze the patterns and implications of mother-child interactions in sports and outdoor activities for health and relationship closeness. However, it is also likely that health, relationship closeness, and perceptions of "good" parenting are overwhelmingly influenced by factors besides motherchild interactions in sports and outdoor activities.

Yet, such mother-child interactions remain meaningful nonetheless and offer insights into why and how families allocate and prioritize their time - and the potential effects that may derive from this. Relatedly, the research may be used to inform practices, policies, and programs that seek to support mother-child interactions in sports and outdoor activities and the potential benefits that they may have for health and relationship closeness. For example, the findings suggest that mother-child interactions in sports and outdoor activities, including organized sports, seem to be generally positive in terms of their implications for meeting parenting role expectations and nurturing health and feelings of relationship closeness for both generations. Thus, support for mother-child interactions in sports and outdoor activities seems warranted. Also, given the relevance of specifically organized sports for children's health and reports of mother-child closeness, and the fact that children's organized sports participation is positively associated with SES, reducing economic barriers for participating in organized sports appears to be important. 
Of course, there are some limitations of the study to recognize. Many of the limitations are due to data constraints. First, it would be preferable to know more about the circumstances under which mother-child interactions in sports and outdoor activities occurred. For example, what sorts of activities were they engaged in? To what extent were they pleasurable? Did they require at least moderate levels of physical activity? How long were the interactions? Second, we wish we could have been able to follow these families over time and better assess the extent to which family interactions around sports and outdoor activities were common or infrequent occurrences, at different stages of the child's life. There is reason, and now data, to explore the changes that the families in our sample experienced into the teenage years (i.e., age 15), for example. However, this focus was beyond the scope of the present study; future work should extend our research and pursue these questions. Relatedly, we would like to have better information about how such interactions may ebb and flow with health statuses and relationship closeness. Furthermore, all of these research pursuits should be applied to understanding diverse family forms. Finally, it would have been ideal to be able to match mother-child behaviors with their voiced perceptions of intensive mothering expectations and purposive leisure pursuits. Additional research should seek to address the shortcomings of the present study and continue to inform our understandings of family-based interactions in sports and outdoor activities.

Nevertheless, this study contributes to research on family-based interactions in sports and outdoor activities. It offers unique quantitative evidence from the U.S. of the patterns, predictors, and implications of these interactions and suggests that mother-child activities are often extensions of intensive mothering and purposive leisure expectations. It also contributes insight into the behaviors and implications of family interactions among more disadvantaged families, who have often been neglected in research on family-based interactions around sports 
and outdoor activities. In fact, the findings suggest that disadvantaged families experience many of the same parenting pressures and benefit from many of the same parent-child interactions as more advantaged families (Author Citation; Kendig and Bianchi, 2008; Sabo and Veliz, 2008).

Overall, the findings of this study reveal that mother-child interactions in sports and outdoor activities are quite common; generally, they occur at least once per week or more. In addition, these interactions seem to be positively associated with self-perceptions of good parenting, mother's health, and mother's perceptions of mother-child relationship closeness. In addition, we find evidence that it is child's organized sports participation that is positively associated with their reports of health and mother-child relationship closeness, in contrast to our assumption that mother-child interactions in sports and outdoor activities would also translate to improved health and relationship closeness, from the child's perspective. Future research should continue to seek to understand family-based patterns of sports and outdoor activities and their implications for health, relationships, and other aspects of life. 


\section{References}

Althoff, T., Sosič, R., Hicks, J. L., King, A. C., Delp, S. L., \& Leskovec, J. 2017. Large-scale physical activity data reveal worldwide activity inequality. Nature, 547(7663), 336-339.

Author Citation.

Bianchi, S. M., Robinson, J. P., \& Milkie, M. A. 2006. Changing rhythms of American family life. New York: Russell Sage.

Biddle, B. 1986. Recent developments in role theory. Annual Review of Sociology 12:67-92.

Booker, C. L., Skew, A. J., Kelly, Y. J., \& Sacker, A. 2015. Media use, sports participation, and well-being in adolescence: Cross-Sectional findings from the UK Household Longitudinal Study. American Journal f Public Health, 105(1), 173-179.

Brooks, S. N., Knudtson, M., \& Smith, I. 2017. Some kids are left behind: The failure of a perspective, using critical race theory to expand the coverage in the sociology of youth sports. Sociology Compass, 11, e12445.

Cairney, J., Joshi, D., Kwan, M., Hay, J., \& Faught, B. 2015. Children's participation in organized sport and physical activities and active free play: Exploring the impact of time, gender and neighbourhood household income using longitudinal data. Sociology of Sport Journal, 32(3), 266-283.

Christopher, K. 2012. Extensive mothering. Gender \& Society 26:73-96.

Coakley, J. 2006. The good father: Parental expectations and youth sports. Leisure Studies, 25(2), 153-163.

Coakley, J. 2011. Youth sports: What counts as “positive development?" Journal of Sport \& Social Issues, 35(3), 306-324.

Craig, Lyn, and Killian Mullan. 2010. Parenthood, Gender and Work-Family Time in the United 
States, Australia, Italy, France, and Denmark. Journal of Marriage and Family 72:13441361.

Dlugonski, Deidre and Motl, Robert W. Motl. 2013. Marital Status and Motherhood:

Implications for Physical Activity, Women \& Health 53:203-215.

Eckstein, R. 2017. How college athletics are hurting girls'sports: The pay-to-play pipeline. Lanham, MD: Rowman \& Littlefield.

Elliott, S., Powell, R., \& Brenton, J. 2013. Being a good mom. Journal of Family Issues 36:351370.

Erkelenz, Nanette, Anja C. Schreiber, Susanne Kobel, Sarah Kettner, Clemens Drenowatz, and Jürgen M. Steinacker. 2014. Relationship of Parental Health-Related Behaviours and Physical Fitness in Girls and Boys. Journal of Public Health 22:407-414.

Goode, W. J. 1960. A theory of role strain. American Sociological Review 25:483-496.

Harrington, M. 2015. Practices and meaning of purposive family leisure among working- and middle-class families. Leisure Studies, 34(4), 471-486.

Haskell, W. L., Blair, S. N., \& Hill, J. O. 2009. Physical activity: Health outcomes and importance for public health policy. Preventive Medicine 49:280-282.

Hays, Sharon. 1996. The Cultural Contradictions of Motherhood. New Haven, CT: Yale University.

Hesketh KR, Goodfellow L, Ekelund U, McMinn AM, Godfrey KM, Inskip HM, Cooper C., Harvey NC, Van Sluijs EMF. 2014. Activity levels in mothers and their preschool children. Pediatrics 133(4):973-980.

Humphreys, B. R., \& Ruseski, J. E. 2013. Physical Activity and Health Outcomes: Evidence from Canada. Health Economics 23:33-54. 
Kahan, D., \& McKenzie, T. L. 2015. The potential and reality of physical education in controlling overweight and obesity. American Journal of Public Health, 105(4), 653659.

Kendig, S. M., \& Bianchi, S. M. 2008. Single, cohabitating, and married mothers' time with children. Journal of Marriage and Family 70:1228-1240.

Knoester, C., Haynie, D. L., \& Stephens, C. M. (2006). Parenting practices and adolescents' friendship networks. Journal of Marriage \& Family, 68(5), 1247-1260.

Knoester, C., \& Petts, R. J. 2017. Fathers' parenting stress after the arrival of a new child. Family Relations 66:367-382.

Lareau, Annette. 2003. Unequal Childhoods: Class, Race and Family Life. Berkeley: University of California Press.

Marsiglio, W., \& Roy, K. 2012. Nurturing dads: Social initiatives for contemporary fatherhood. New York: Russell Sage Foundation.

Messner, M. A. 2009. It's all for the kids: Gender, families, and youth sports. Berkeley: University of California Press.

Messner, M. A., \& Musto, M. 2014. Where are the kids?. Sociology of Sport Journal, 31(1), 102-122.

Milkie, M. A., Nomaguchi, K. M., \& Denny, K. E. 2015. Does the amount of time mothers spend with children or adolescents matter? Journal of Marriage and Family 77:355372.

Montez de Oca, J. 2005. “As our muscles get softer, our missile race becomes harder”: Cultural citizenship and the "Muscle Gap.” Journal of Historical Sociology, 18(3), 145-172.

Phares, V., Fields, S., \& Kamboukos, D. 2009. Fathers' and mothers' involvement with their 
adolescents. Journal of Child and Family Studies, 18(1):1-9.

Physical Activity Council. 2016. 2016 Participation Report: The Physical Activity Council's annual study tracking sports, fitness, and recreation participation in the U.S. Overview Report from the Physical Activity Council.

Project Play. 2015. Sport for All, Play for Life. Aspen Institute Sports \& Society Program Report.

Project Play. 2016. State of play: 2016. Aspen Institute Sports \& Society Program Report. Raley, S., Bianchi, S. M., \& Wang, W. (2012). When do fathers care? Mothers' economic contribution and fathers' involvement in child care. American Journal of Sociology, 117(5), 1422-1459.

Ramraj, C., Shahidi, F. V., Jr.Darity, W., Kawachi, I., Zuberi, D., \& Siddiqi, A. 2016. Equally inequitable? A cross-national comparative study of racial health inequalities in the United States and Canada. Social Science \& Medicine, 161, 19-26.

Reichman, N., Teitler, J., Garfinkel, I., \& McLanahan, S. 2001. Fragile families: Sample and design. Children and Youth Services Review, 23, 303-326.

Rhodes, R. E., Berry, T., Craig, C. L., Faulkner, G., Latimer-Cheung, A., Spence, J. C., \& Tremblay, M. S. 2013. Understanding parental support of child physical activity behavior. American Journal of Health Behavior, 37(4), 469-477.

Rhodes, Ryan E., and Clarise Lim. 2018. Promoting Parent and Child Physical Activity Together: Elicitation of Potential Intervention Targets and Preferences. Health Education \& Behavior 45:112-123.

Risman, B. J. (1998). Gender vertigo: American families in transition. New Haven: Yale University Press. 
Sabo, Don, \& Veliz, Phil. 2008. Go out and play: Youth sports in America. East Meadow, NY: Women's Sports Foundation.

Shaw, S. M., \& Dawson, D. 2001. Purposive leisure: Examining parental discourses on family activities. Leisure Sciences, 23(4), 217-231.

Solomon-Moore, E., Toumpakari, Z., Sebire, S. J., Thompson, J. L., Lawlor, D. A., \& Jago, R. 2018. Roles of mothers and fathers in supporting child physical activity: A crosssectional mixed-methods study. BMJ Open 8:1-10.

Stone, Pamela. 2007 Opting Out?: Why Women Really Quit Careers and Head Home. Berkeley: University of California Press.

Thompson, S. M. 1999. Mother's Taxi: Sport and women's labor. Albany: State University of New York Press.

Wheeler, S., \& Green, K. 2014. Parenting in relation to children's sports participation: generational changes and potential implications. Leisure Studies, 33(3), 267-284. 
Table 1. Descriptive Statistics

Mor $\% \quad S D \quad$ Min $\quad$ Max

Mother-Child Sports or Outdoor Activities

1.92

3.05

Mother's Report of Being a Good Parent

3.56

4.10

3.71

3.67

1.21

0.72

Mother's Health

1.03

Child's Health

Mother-Child Closeness (Mother report)

Mother-Child Closeness (Child report)

Background Characteristics

Mother's Age

High School Education $^{\text {a }}$

Some College ${ }^{\mathrm{a}}$

College or more ${ }^{a}$

0.97

0.56

0.70

$0 \quad 4$

Income

Black $^{\mathrm{b}}$

Latina $^{\mathrm{b}}$

Cohabiting with Father ${ }^{\mathrm{c}}$

Living Apart from Father ${ }^{\mathrm{c}}$

New Partner

Female Child

Child's Organized Sports Participation

Father-Child Sports or Outdoor Activities

Number of Children

Mother's Health (W4)

\begin{tabular}{rlrr}
34.52 & 6.01 & 23 & 56 \\
$21 \%$ & - & 0 & 1 \\
$42 \%$ & - & 0 & 1 \\
$17 \%$ & - & 0 & 1 \\
4.66 & 5.12 & 0 & 90 \\
$49 \%$ & - & 0 & 1 \\
$26 \%$ & - & 0 & 1 \\
$10 \%$ & - & 0 & 1 \\
$59 \%$ & - & 0 & 1 \\
$20 \%$ & - & 0 & 1 \\
$47 \%$ & - & 0 & 1 \\
$50 \%$ & - & 0 & 1 \\
1.67 & 1.32 & 0 & 4 \\
2.93 & 1.34 & 1 & 6 \\
3.65 & 1.02 & 1 & 5 \\
4.48 & 0.75 & 1 & 5 \\
\hline
\end{tabular}

$\mathrm{N}=3252$

aess than high school is used as reference category, ${ }^{\mathrm{b}}$ White/Other is used as reference category, ${ }^{c}$ Married to father is used as reference category. 
Table 2. Results from OLS Regressions Predicting Mother's Report of Being a Good Parent

\begin{tabular}{|c|c|c|}
\hline & \multicolumn{2}{|c|}{ Parent Self-Rating } \\
\hline Variable & $b$ & $S E$ \\
\hline Mother-Child Sports or Outdoor Activities & $0.06^{* * *}$ & 0.01 \\
\hline Mother's Age & $0.01^{*}$ & 0.00 \\
\hline High School Education ${ }^{\mathrm{a}}$ & -0.03 & 0.04 \\
\hline Some College ${ }^{\mathrm{a}}$ & $-0.13^{* * *}$ & 0.03 \\
\hline College or more ${ }^{\mathrm{a}}$ & $-0.13^{* *}$ & 0.05 \\
\hline Income & 0.00 & 0.00 \\
\hline Black $^{b}$ & $0.27^{* * *}$ & 0.03 \\
\hline Latina $^{\mathrm{b}}$ & $0.11^{* *}$ & 0.04 \\
\hline Cohabiting with Father ${ }^{c}$ & 0.05 & 0.05 \\
\hline Living Apart from Father ${ }^{\mathrm{c}}$ & $0.08^{*}$ & 0.04 \\
\hline New Partner & -0.03 & 0.04 \\
\hline Female Child & -0.00 & 0.02 \\
\hline Child's Organized Sports Participation & 0.03 & 0.03 \\
\hline Father-Child Sports or Outdoor Activities & $0.02^{* *}$ & 0.01 \\
\hline Number of Children & -0.01 & 0.01 \\
\hline Mother's Health (W4) & $0.08^{* * * *}$ & 0.01 \\
\hline Child's Health (W4) & $0.08^{* * * *}$ & 0.02 \\
\hline$R^{2}$ & \multicolumn{2}{|c|}{0.06} \\
\hline
\end{tabular}

$\mathrm{N}=3252,{ }^{+} p<.10 .{ }^{*} p<.05 . * * p<.01 .{ }^{* * *} p<.001$.

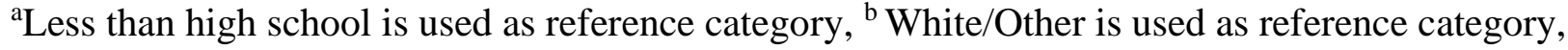

${ }^{\mathrm{c}}$ Married to father is used as reference category. 
Table 3. Results from OLS and Logistic Regressions Predicting Mother-Child Sports and Outdoor Activities and Child's Organized Sports Participation

\begin{tabular}{|c|c|c|c|c|}
\hline \multirow[b]{2}{*}{ Variable } & \multicolumn{2}{|c|}{$\begin{array}{l}\text { Mother-Child } \\
\text { Interactions }\end{array}$} & \multicolumn{2}{|c|}{$\begin{array}{c}\text { Organized Sports } \\
\text { Participation } \\
\end{array}$} \\
\hline & $b$ & $S E$ & OR & $S E$ \\
\hline Mother's Age & $-0.02^{* * * *}$ & 0.00 & 1.00 & 0.01 \\
\hline High School Education $^{\mathrm{a}}$ & -0.06 & 0.07 & 1.10 & 0.13 \\
\hline Some College ${ }^{\mathrm{a}}$ & -0.02 & 0.06 & $1.45^{* * *}$ & 0.15 \\
\hline College or more ${ }^{\mathrm{a}}$ & 0.07 & 0.08 & $1.97^{* * *}$ & 0.29 \\
\hline Income & -0.00 & 0.01 & $1.07^{* * *}$ & 0.01 \\
\hline Black $^{b}$ & $-0.35^{* * *}$ & 0.06 & 0.84 & 0.09 \\
\hline Latina $^{b}$ & $-0.29^{* * *}$ & 0.06 & $0.73^{* *}$ & 0.08 \\
\hline Cohabiting with Father ${ }^{\mathrm{c}}$ & -0.00 & 0.08 & 0.83 & 0.12 \\
\hline Living Apart from Father ${ }^{\mathrm{c}}$ & $0.19^{* *}$ & 0.06 & 1.06 & 0.12 \\
\hline New Partner & $-0.20^{* *}$ & 0.06 & $0.76^{*}$ & 0.09 \\
\hline Female Child & $0.13^{* *}$ & 0.04 & $0.56^{* * *}$ & 0.04 \\
\hline Child's Organized Sports Participation & $0.29^{* * * *}$ & 0.04 & - & - \\
\hline Father-Child Sports or Outdoor Activities & $0.14^{* * * *}$ & 0.02 & $1.15^{* * *}$ & 0.04 \\
\hline Number of Children & $0.03^{*}$ & 0.02 & $0.87^{* * *}$ & 0.03 \\
\hline Mother's Health (W4) & $0.05^{*}$ & 0.02 & $1.16^{* * *}$ & 0.05 \\
\hline Child's Health (W4) & 0.05 & 0.03 & 1.00 & 0.06 \\
\hline$R^{2}$ & \multicolumn{2}{|c|}{0.07} & \multicolumn{2}{|c|}{$0.09^{\mathrm{d}}$} \\
\hline
\end{tabular}

$\mathrm{N}=3252,{ }^{+} p<.10 .{ }^{*} p<.05 .{ }^{*} * p<.01 . * * * p<.001$.

a Less than high school is used as reference category, ${ }^{\mathrm{b}}$ White/Other is used as reference category, ${ }^{\mathrm{c}}$ Married to father is used as reference category, ${ }^{\mathrm{d}}$ Pseudo- $\mathrm{R}^{2}$ reported for logistic regression. 
Table 4. Results from OLS Regressions Predicting Mother's and Child's Overall Health

\begin{tabular}{|c|c|c|c|c|}
\hline \multirow[b]{2}{*}{ Variable } & \multicolumn{2}{|c|}{ Mother's Health } & \multicolumn{2}{|c|}{ Child's Health } \\
\hline & $b$ & $S E$ & $b$ & $S E$ \\
\hline Mother-Child Sports or Outdoor Activities & $0.03^{*}$ & 0.01 & -0.02 & 0.02 \\
\hline Mother's Age & -0.00 & 0.00 & -0.01 & 0.02 \\
\hline High School Education ${ }^{\mathrm{a}}$ & -0.02 & 0.05 & -0.01 & 0.06 \\
\hline Some College ${ }^{\mathrm{a}}$ & 0.03 & 0.05 & 0.04 & 0.05 \\
\hline College or more ${ }^{\mathrm{a}}$ & 0.10 & 0.06 & 0.09 & 0.07 \\
\hline Income & $0.02^{* * * *}$ & 0.00 & -0.00 & 0.00 \\
\hline Black b & -0.01 & 0.04 & -0.04 & 0.05 \\
\hline Latina $^{\mathrm{b}}$ & 0.03 & 0.05 & $-0.15^{*}$ & 0.06 \\
\hline Cohabiting with Father ${ }^{\mathrm{c}}$ & -0.09 & 0.06 & -0.03 & 0.07 \\
\hline Living Apart from Father ${ }^{\mathrm{c}}$ & 0.02 & 0.05 & -0.02 & 0.05 \\
\hline New Partner & -0.02 & 0.05 & 0.04 & 0.05 \\
\hline Female Child & $0.06^{+}$ & 0.03 & -0.01 & 0.04 \\
\hline Child's Organized Sports Participation & 0.05 & 0.03 & $0.13^{* *}$ & 0.04 \\
\hline Father-Child Sports or Outdoor Activities & $0.03^{+}$ & 0.01 & 0.02 & 0.02 \\
\hline Number of Children & $-0.03^{* *}$ & 0.01 & 0.01 & 0.01 \\
\hline Mother's Health (W4) & $0.42^{* * *}$ & 0.02 & 0.01 & 0.02 \\
\hline Child's Health (W4) & $0.09^{* * * *}$ & 0.02 & $0.05^{*}$ & 0.02 \\
\hline$R^{2}$ & & & & 02 \\
\hline
\end{tabular}

$\mathrm{N}=3252,{ }^{+} p<.10 .{ }^{*} p<.05 .{ }^{* *} p<.01 .{ }^{* * *} p<.001$.

${ }^{a}$ Less than high school is used as reference category, ${ }^{b}$ White/Other is used as reference category,

${ }^{c}$ Married to father is used as reference category. 
Table 5. Results from OLS Regressions Predicting Mother-Child Relationship Closeness

\begin{tabular}{|c|c|c|c|c|}
\hline \multirow[b]{2}{*}{ Variable } & \multicolumn{2}{|c|}{ Mother's Reports } & \multicolumn{2}{|c|}{ Child's Reports } \\
\hline & $b$ & $S E$ & $b$ & $S E$ \\
\hline Mother-Child Sports or Outdoor Activities & $0.04^{* * * *}$ & 0.01 & -0.00 & 0.01 \\
\hline Mother's Age & $0.00^{*}$ & 0.00 & 0.00 & 0.00 \\
\hline High School Education ${ }^{\mathrm{a}}$ & $0.08^{*}$ & 0.03 & -0.01 & 0.04 \\
\hline Some College ${ }^{\mathrm{a}}$ & 0.05 & 0.03 & -0.01 & 0.04 \\
\hline College or more ${ }^{a}$ & 0.01 & 0.04 & 0.04 & 0.05 \\
\hline Income & -0.00 & 0.00 & -0.00 & 0.00 \\
\hline Black $^{b}$ & $0.08^{* *}$ & 0.03 & $0.08^{*}$ & 0.03 \\
\hline Latina $^{\mathrm{b}}$ & -0.05 & 0.03 & 0.04 & 0.04 \\
\hline Cohabiting with Father ${ }^{c}$ & -0.06 & 0.04 & $-0.12^{*}$ & 0.05 \\
\hline Living Apart from Father ${ }^{\mathrm{c}}$ & $0.07^{*}$ & 0.03 & -0.01 & 0.04 \\
\hline New Partner & -0.03 & 0.03 & 0.05 & 0.04 \\
\hline Female Child & 0.01 & 0.02 & $0.08^{* *}$ & 0.03 \\
\hline Child's Organized Sports Participation & $0.05^{*}$ & 0.02 & $0.08^{* *}$ & 0.03 \\
\hline Father-Child Sports or Outdoor Activities & $0.02^{*}$ & 0.01 & $0.02^{+}$ & 0.01 \\
\hline Number of Children & $-0.03^{* * *}$ & 0.01 & -0.02 & 0.01 \\
\hline Mother's Health (W4) & $0.03^{* *}$ & 0.01 & 0.03 & 0.02 \\
\hline Child's Health (W4) & $0.04^{* *}$ & 0.01 & 0.06 & 0.03 \\
\hline$R^{2}$ & \multicolumn{2}{|c|}{0.05} & \multicolumn{2}{|c|}{0.02} \\
\hline
\end{tabular}

$\mathrm{N}=3252,{ }^{+} p<.10 .{ }^{*} p<.05 . * * p<.01 .{ }^{* * *} p<.001$.

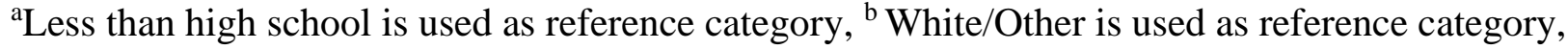

${ }^{c}$ Married to father is used as reference category. 